\section{Rol de la enfermera en el anciano con cáncer. Revisión bibliográfica}

\section{Role of the nurse in the elderly with cancer. Bibliographic review}

Mercedes Montoro-Lorite ${ }^{1, *}$ Montserrat Canalias-Reverter ${ }^{2}$ Montserrat Valverde-Bosch ${ }^{3}$ Sergi Font-Ritort ${ }^{4}$

1. Enfermera. Hospital de día de Oncología. Instituto Clínic de Enfermedades Hematológicas y Oncológicas. Hospital Clínic de Barcelona. Barcelona. España.

2. Responsable enfermera. Instituto de Medicina y Dermatología. Hospital Clínic de Barcelona. Barcelona. España.

3. Responsable enfermera. Instituto Clínic de Enfermedades Hematológicas y Oncológicas. Hospital Clínic de Barcelona. Barcelona. España.

4. Unidad Funcional Interdisciplinaria Sociosanitaria (UFISS). Unidad de Cuidados Paliativos. Hospital de Mataró. Mataró. Barcelona. España.

*Autor para correspondencia.

Correo electrónico:MMONTORO@clinic.cat (Mercedes Montoro-Lorite).

Recibido el 25 de septiembre de 2018; aceptado el 14 de marzo de 2019

\section{RESUMEN}

El aumento de la incidencia y prevalencia de procesos cancerígenos en los ancianos representa un nuevo reto para las políticas sanitarias y sociales. El presente artículo reflexiona sobre la necesidad de un modelo de atención integral, integrada y multidimensional para el cuidado del anciano con cáncer y su familia. El objetivo principal es identificar, recopilar, analizar y sintetizar la literatura científica más actual y relevante sobre las funciones y/o rol de la enfermera en el cuidado del anciano con cáncer. Material y método: se realizó una revisión bibliográfica en PubMed (Medline), Cochrane Library Plus, Dialnet y ScienceDirect ${ }^{\circ}$ (Elsevier), entre enero de 2007 y diciembre de 2017, de la literatura científica sobre las funciones y/o rol de la enfermera en el cuidado del anciano con cáncer. Los estudios fueron seleccionados por revisores pares de forma independiente utilizando el instrumento PRISMA. Resultados: Se identificaron 28 estudios que cumplieron los criterios de inclusión. Toda la bibliografía revisada recomienda el desarrollo e implementación de programas de atención individualizados como el pilar principal de atención al anciano con cáncer. En ellos, las enfermeras desempeñan un papel clave en la gestión y coordinación durante todo el proceso de atención.

Conclusiones: El modelo de cuidado de los ancianos con cáncer debe abarcar todo el proceso de atención: prevención, detección precoz, evaluación, tratamiento, educación e investigación. Es esencial el rol de la enfermera como gestora, coordinadora, educadora y cuidadora en la atención al anciano con cáncer.

PALABRAS CLAVE: Evaluación geriátrica, enfermería oncológica.

\section{ABSTRACT}

The increase in the incidence and prevalence of carcinogenic processes in the elderly represents a new challenge for health and social policies. This article reflects on the need for a comprehensive, integrated and multidimensional care model for the care of the elderly with cancer and his family. The main objective is to identify, compile, analyse and synthesize the most current and relevant scientific literature on the roles and/or role of the nurse in the care of the elderly person with cancer. Material and method: a bibliographic review was made in PubMed (Medline), Cochrane Library Plus, Dialnet and ScienceDirect ${ }^{\circ}$ (Elsevier) between January 2007 and December 2017 of the literature on the functions and/or roles of the nurse in the care of the elderly with cancer. The studies were selected by peer reviewers independently using the PRISMA instrument. Results: Twenty-eight studies that met the inclusion criteria were identified. All the revised literature recommends the development and implementation of individualized care programs as the main pillar of care for the elderly with cancer. In them, nurses play a key role in the management and coordination throughout the entire care process. Conclusions: The model of the care of the elderly with cancer must cover the whole process of attention: prevention, early detection, evaluation, treatment, education and research. The role of the nurse is essential as a manager, coordinator, educator and caregiver in the care of the elderly with cancer.

KEYWORDS: Geriatric Assessment, Oncology nursing.

\section{- INTRODUCCIÓN}

Con el envejecimiento de la población y el aumento constante de la incidencia de cáncer en las personas de edad avanzada, el abordaje de los ancianos con cáncer se ha convertido en un importante problema de salud pública a nivel mundial ${ }^{1}$. El envejecimiento es el principal factor de riesgo intrínseco para el desarrollo del cáncer². En España se estima que el $60 \%$ de las personas que sufren cáncer tiene más de 65 ańos y en igual porcentaje los supervivientes de cáncer ${ }^{3}$.

El cuidado de los ancianos con cáncer requiere de una atención integral y coordinada que aborde aspectos físicos, psicológicos y sociales. Para poder identificar y desarrollar un plan de atención individualizado e integral al anciano con cáncer es preciso la integración de la experiencia de los distintos profesionales de la salud: enfermeras, farmacéuticos, 
geriatras, oncólogos, cirujanos, radioterapeutas, fisioterapeutas, dietistas, trabajadores sociales, etc. en un equipo multidisciplinar. Las enfermeras especialistas en geriatría tienen un papel fundamental en estos equipos, ya que sus conocimientos especializados en envejecimiento les permiten comprender cómo este se entrelaza con la atención al cáncer, fusionando el arte y la ciencia de la enfermería geriátrica con el arte y la ciencia de la enfermería oncológica ${ }^{4}$. Janine Overcash describió las funciones de las enfermeras en estos equipos: coordinar y recopilar la información, inspirar el diálogo, fomentar la cooperación para activar un plan de cuidado individualizado, seguimiento activo, actualización constante y planificación de los cambios necesarios en el plan de atención e informar de ellos al resto del equipo5.

Es internacionalmente reconocida la figura de la enfermera de oncología geriátrica (EOG), esencial para identificar el mejor marco de atención y de cuidados ${ }^{6}$. Diversos estudios internacionales han analizado los beneficios de incorporar su figura en unidades de oncología, destacando una mayor satisfacción y mejor calidad de vida del paciente $^{7,8}$, diminución del uso de pruebas diagnósticas ${ }^{8}$, mayor comprensión de la educación realizada ${ }^{9}$ y reducción de la sintomatología secundaria a la quimioterapia ${ }^{10,11}$. En Espańa no se han desarrollado ni implementado de forma unificada los roles de la EOG en todo el territorio. Cada unidad o servicio de oncología ha desarrollado su propio método, en función de sus recursos personales, económicos y el tipo de población que atienden ${ }^{12}$. En enero 2017, la agencia de calidad sanitaria de Andalucía presentó un manual de competencias profesional para las enfermeras de práctica avanzada en cuidados oncológicos ${ }^{13}$ que incluye la competencia en coordinación de cuidados complejos, como es el caso de los ancianos con cáncer. En la tabla 1 se exponen las competencias profesionales para enfermería en atención al anciano con cáncer.

Este estudio se inició con el objetivo de identificar, recopilar, analizar y sintetizar la literatura científica más actual y relevante sobre las funciones y/o rol de la enfermera en el cuidado del anciano con cáncer.
Considerando el propósito general de este estudio, la revisión se centra en dos perspectivas principales que son los objetivos específicos:

1. Definir los modelos internacionales de atención de enfermería al anciano con cáncer.

2. Describir el rol de la enfermera en el continuum del cuidado del anciano con cáncer.

\section{- MATERIAL Y MÉTODOS}

Se realizó una revisión bibliográfica siguiendo el método PRISMA de ensayos controlados aleatorizados, revisiones sistemáticas, estudios observacionales y revisiones integradoras en diferentes bases de datos. Se admitieron artículos en inglés y español publicados desde enero de 2007 hasta diciembre 2017, que incluyeran modelos de atención de enfermería al anciano con cáncer y las funciones y/o rol de la enfermera en el cuidado del anciano con cáncer. Se excluyeron los estudios que no especificaban el perfil de la enfermera en el cuidado del anciano con cáncer. También se incluyeron estudios adicionales que fueron identificados por la búsqueda manual en listas de referencias. Dos autores, de forma independiente, se encargaron de la selección de los artículos incluidos, la extracción y el análisis de los datos.

\section{- RESULTADOS}

La búsqueda se realizó e1 1 de diciembre de 2017 en las siguientes bases de datos: Medline (PubMed), Cochrane Library Plus, Dialnet y ScienceDirect, y se obtuvo un total de 186 publicaciones. Las palabras clave utilizadas fueron: Geriatric Assessment [Mesh] and Oncology nursing [Mesh]. Cinco publicaciones adicionales se identificaron durante la bús-

Tabla 1. Lista de competencias profesionales para enfermería en atención al anciano con cáncer ${ }^{\mathrm{a}}$

- Gestión y coordinación del proceso diagnóstico del anciano con cáncer

- Atención personalizada durante el proceso diagnóstico del anciano con cáncer

- Valoración e identificación de las necesidades del anciano de forma integral y establecer un plan de intervención individualizado

- Identificación, valoración y atención a las necesidades psicológicas, espirituales, familiares y sociales como parte de la valoración total de la unidad anciano-familia

- Atención que integre las necesidades y deseos de la persona, potenciando su autonomía y movilizando los apoyos necesarios para lograr su adaptación efectiva y positiva

- Valorar y atender las necesidades del cuidador de forma personalizada, realizando un abordaje integral y contribuir al saludable desempeño de su rol

- Identificación de las capacidades funcionales del anciano con cáncer en el manejo de su situación de salud

- Realización y seguimiento de un plan de intervención individualizado

- Coordinación y participación en grupos de trabajo relacionados con el diseño, la implantación, desarrollo o seguimiento de procesos asistenciales o de soporte y/o planes integrales en su ámbito asistencial

- Planificación conjunta del alta hospitalaria entre los distintos profesionales de salud implicados en la atención del anciano con cáncer

- Seguimiento conjunto del plan de actuación del anciano con cáncer que requiere abordaje de recursos al alta hospitalaria.

- Garantizar la continuidad asistencial a lo largo de la atención que recibe el anciano con cáncer

aAdaptado del Manual de Competencias Profesionales Enfermería de Práctica en Cuidados Oncológicos ${ }^{13}$. 
queda. Después de la comprobación de duplicados fueron excluidas 88 . Un análisis cuidadoso de los títulos, resúmenes y referencias se tradujo en 45 documentos de texto completo que fueron seleccionados para su inclusión. En total, 28 estudios cumplieron los criterios de inclusión, y se incluyeron en la revisión. La estrategia de búsqueda se encuentra representada en la figura 1 .

\section{Modelos de atención de enfermería al anciano con cáncer}

Existen distintos modelos de atención de enfermería en oncología geriátrica. En ellos, las enfermeras desempeñan un papel clave en la gestión y coordinación del programa de atención individualizado del anciano con cáncer ${ }^{4,14}$ :

- En Francia existe el grado académico de especialidad de enfermería en oncología geriátrica ${ }^{4}$. Las enfermeras formadas en esta especialidad están capacitadas para gestionar y coordinar el programa de atención en oncología geriátrica, que incluyen los distintos roles de enfermería en el ámbito asistencial, de investigación y de educación. Tienen formación específica para crear y administrar planes de actuación individualizados a los ancianos con cáncer y asegurar el cumplimiento de estos en los diferentes entornos asistenciales 5 .

- Australia incluye tres enfoques de políticas clínicas en oncología geriátrica. Los tres modelos coinciden en incluir una enfermera consultora en oncología geriátrica. Su principal función es evaluar a los ancianos con cáncer antes de la consulta médica e identificar aquellos que precisaran apoyo durante el tratamiento del cáncer ${ }^{4,5}$.

- En Estados Unidos existe la figura de la enfermera practicante en oncología geriátrica, encargada de evaluar y desarrollar un plan de cuidados individualizado a cada anciano con cáncer. El ámbito de desarrollo de sus funciones varía según los proyectos de investigación. En el Memorial Sloan Kettering Cancer Center realiza en la unidad de hospitalización un equipo consultor de enfermería, y en el University of Rochester Medical Center James P. Wilmot se combina una consulta ambulatoria de oncología geriátrica con otra de oncología primaria ${ }^{4,5,15}$.

\section{Rol de enfermera en el continuum del cuidado del anciano con cáncer}

El objetivo principal de las enfermeras en oncología y geriatría es maximizar la independencia del anciano, evaluando y abordando las áreas clave en las que estos pueden presentar déficits reales o potenciales, basándose en un enfoque holístico que permita proporcionar una atención continua que incluya la prevención, detección precoz, evaluación, tratamiento y seguimiento del anciano con cáncer ${ }^{6}$.

\section{PREVENCIÓN Y PROMOCIÓN DE LA SALUD}

Entre el $80 \%$ y el $90 \%$ de los procesos cancerígenos son prevenibles ${ }^{16}$. Las EOG deben aplicar estrategias de promoción de la salud con intervenciones educativas para los principales factores de riesgo de cáncer modificables como son: el tabaco, la dieta, el alcohol, el sedentarismo, la exposición ocupacional y ambiental y las infecciones crónicas.

\section{CRIBADO DE CÁNCER}

A nivel europeo, en España encontramos programas de cribado de cáncer, pero se ha detectado que los ancianos no se benefician de estos pro-

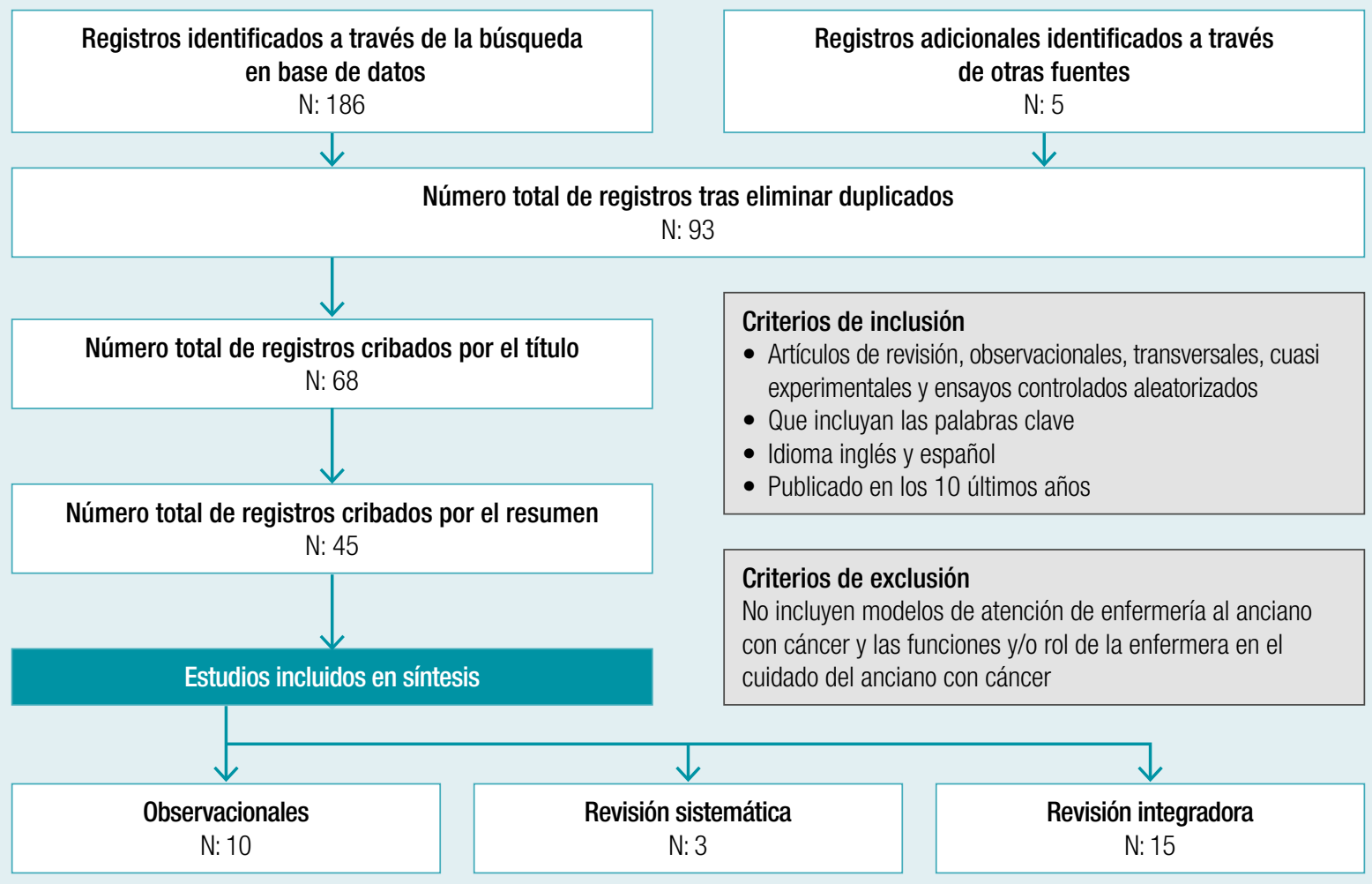

Figura 1. Diagrama de flujo del proceso de búsqueda y selección. 
gramas $^{3,17}$. Las EOG deben implementar estrategias de asesoramiento para los ancianos y su familia, explicando los riesgos y complicaciones de las pruebas diagnósticas que facilitará la toma de decisiones con respecto al cribado del cáncer.

\section{Diagnóstico y EVALUACión}

Tras la detección y el diagnóstico, es necesario realizar una evaluación para determinar el mejor tratamiento para el anciano. Diversos estudios han demostrado que la edad biológica no es el mejor predictor de la tolerancia al tratamiento ${ }^{18-21}$. La evaluación del anciano con cáncer utilizando la valoración geriátrica integral (VGI) permite adaptar mejor el tratamiento, 1,6,20,21. La VGI es el instrumento más completo y la principal herramienta para la detección de los riesgos presentes o potenciales a nivel físico, emocional, cognitivo, funcional y problemas psicosociales que pueden afectar al tratamiento del cáncer ${ }^{6}$. La VGI permite conocer el estado de salud basal inicial y monitorizar su evolución a lo largo de la enfermedad ${ }^{4}$. No existe acuerdo sobre qué escalas deben formar parte de la VGI para el anciano con cáncer; sí la recomendación de que debe incluir una evaluación cognitiva, funcional y psicosocial a través de escalas específicas ${ }^{21-23}$. En la tabla 2 se encuentran descritas las herramientas más utilizadas en la VGI por las EOG para la evaluación del anciano con cáncer. La VGI precisa de mucho tiempo para su administración y de un equipo multidisciplinar para su implementación ${ }^{21-23}$. Se han creado varios instrumentos de cribado para detectar a los ancianos que se puedan beneficiar de una futura VGI y también se han desarrollado instrumentos intermedios entre las escalas de cribado y la VGI, denominadas valoraciones multidimensionales geriátricas, que son más breves y rápidas de administrar ${ }^{3,23}$. Estas herramientas están descritas en la tabla 3.

La evidencia científica ha identificado a las EOG como un gran activo en la evaluación inicial de los ancianos con cáncer, ya sea en consultas propias o en equipos de soporte hospitalario. Estas son un recurso central para recoger y sintetizar toda la información precisa para realizar de forma correcta la $\mathrm{VGI}^{5,6}$.

Tabla 2. Herramientas incluidas en la valoración geriátrica integrala

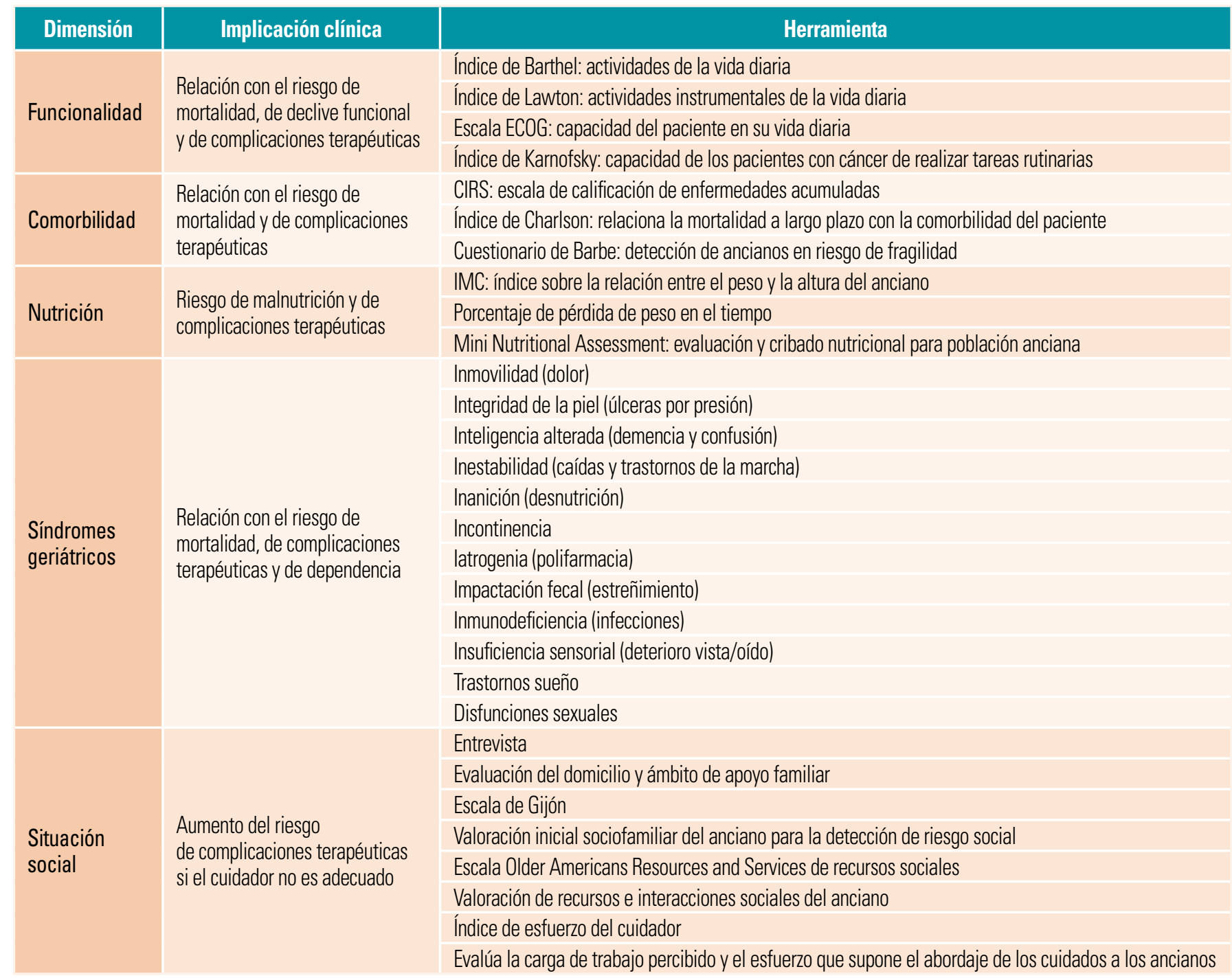

${ }^{a}$ Adaptado de las herramientas recomendadas por la International Society of Geriatric Oncology (SIOG) y los modelos de atención al anciano con cáncer españoles²124.

CIRS: Cumulative Illness Rating Scale; ECOG: Eastern Cooperative Oncology Group. 
Tabla 3. Instrumentos de cribado para ancianos con cáncer y escalas de valoración multidimensional geriátricas

\begin{tabular}{|c|c|c|c|c|}
\hline & Descripción & $\begin{array}{c}\text { Tiempo de } \\
\text { administración }\end{array}$ & Ámbito & Propiedades psicométricas \\
\hline \multicolumn{5}{|c|}{ Instrumentos de cribado } \\
\hline $\begin{array}{l}\text { Elderly Survey }{ }^{25} \\
\text { (VES13) }\end{array}$ & $\begin{array}{l}\text { Es una herramienta simple para identificar ancianos vulnerables en } \\
\text { la comunidad } \\
\text { Formada por } 13 \text { ítems que evalúan la edad, la autoevaluación de } \\
\text { la salud, las limitaciones de la función física y las discapacidades } \\
\text { funcionales } \\
\text { Si la puntuación es igual o mayor de } 3 \text { clasifica al anciano como } \\
\text { vulnerable }\end{array}$ & $5 \min$ & $\begin{array}{c}\text { Comunidad } \\
\text { Hospitalización } \\
\text { Autoevaluación }\end{array}$ & $\begin{array}{l}\text { VES } 13 \text { comparada con VGI } \\
\text { Fiabilidad 0,9 Alfa de Cronbach } \\
\text { Sensibilidad: 87,3\% (IC 95\%: 80,5-92,4) } \\
\text { Especificidad: } 62 \% \text { (IC 95\%: 56,2-67,8) }\end{array}$ \\
\hline ONCODAGE ${ }^{26}(\mathrm{G} 8)$ & $\begin{array}{l}\text { El cuestionario G8 es una herramienta de evaluación geriátrica que } \\
\text { permite a los oncólogos identificar, entre pacientes ancianos con } \\
\text { cáncer, aquellos que deberían beneficiarse de una evaluación } \\
\text { geriátrica exhaustiva } \\
\text { Formada por } 8 \text { ítems que evalúan edad, cambios en el apetito, } \\
\text { pérdida de peso, movilidad, trastorno cognitivo, índice de } \\
\text { masa corporal, polifarmacia y autocontrol y comorbilidades. La } \\
\text { puntuación total es 0-17. Una puntuación menor o igual a } 14 \\
\text { revela vulnerabilidad o fragilidad en el anciano }\end{array}$ & $4 \min$ & $\begin{array}{c}\text { Comunidad } \\
\text { Hospitalización }\end{array}$ & $\begin{array}{l}\text { G8 comparada con VES } 13 \\
\text { Fiabilidad: 0,96 Índice de Kappa } \\
\text { Sensibilidad: 76,5\% (IC 95\%: 73,9-78,9) } \\
\text { Especificidad: } 64.4 \% \text { (IC 95\%: 58,6- } \\
\text { 70,0) }\end{array}$ \\
\hline $\begin{array}{l}\text { Abbreviated } \\
\text { Comprehensive } \\
\text { Geriatric } \\
\text { Assessment }{ }^{27} \text { (aCGA) }\end{array}$ & $\begin{array}{l}\text { Herramienta de evaluación geriátrica que incluye los elementos } \\
\text { más predictivos de vulnerabilidad incluidos en la VGI } \\
\text { Formada por } 15 \text { ítems que evalúan el deterioro global, } \\
\text { las actividades básicas de la vida diaria, las actividades } \\
\text { instrumentales de la vida diaria y el estado mental a través del } \\
\text { Mini Mental }\end{array}$ & $5 \min$ & Hospitalización & $\begin{array}{l}\text { aCGA comparada con VGI } \\
\text { Fiabilidad 0,94 Alfa de Cronbach } \\
\text { Sensibilidad: no evaluada } \\
\text { Especificidad: no evaluada }\end{array}$ \\
\hline $\begin{array}{l}\text { Physical Performance } \\
\text { Test }^{28} \text { (PPT) }\end{array}$ & $\begin{array}{l}\text { Prueba de rendimiento físico de } 7 \text { elementos } \\
\text { Se basa en la observación directa del rendimiento del paciente en } \\
\text { tareas cronometradas. Estas tareas imitan las actividades de la } \\
\text { vida diaria e involucran diferentes niveles de habilidad }\end{array}$ & $\begin{array}{c}\text { Versión breve } \\
\text { de } 9 \text { elementos } \\
\text { (4 min) }\end{array}$ & Hospitalización & $\begin{array}{l}\text { PPT comparada con Karnofsky } \\
\text { Performance Scale } \\
\text { Fiabilidad } \\
\text { Correlación de Pearson }=0,27 ; p<0,001\end{array}$ \\
\hline $\begin{array}{l}\text { Groningen Frailty } \\
\text { Index }^{29}(\mathrm{GFI})\end{array}$ & $\begin{array}{l}\text { Instrumento para la detección de fragilidad para geriatría } \\
\text { Formado por } 15 \text { elementos que evalúan } 4 \text { dominios: dominio } \\
\text { físico (funciones de movilidad, comorbilidad, fatiga física, visión, } \\
\text { audición), cognitivo (deterioro cognitivo), social (apoyo emocional) y } \\
\text { psicológica (estado de ánimo deprimido y sentimientos de ansiedad) } \\
\text { La puntuación total es de } 0 \text { a } 15 \text {. Una puntuación de } 4 \text { o superior } \\
\text { representa fragilidad de moderada a severa }\end{array}$ & $10 \mathrm{~min}$ & $\begin{array}{c}\text { Comunidad } \\
\text { Hospitalización }\end{array}$ & $\begin{array}{l}\text { GFI comparada con G8 } \\
\text { Fiabilidad: 0,87 Alfa de Cronbach } \\
\text { Sensibilidad: } 66 \% \text { (IC 95\%: 56-75) } \\
\text { Especificidad: } 87 \% \text { (IC 85\%: 76-94) }\end{array}$ \\
\hline
\end{tabular}

\section{Escalas de valoración multidimensional geriátricas}

Senior Adult

Oncology Program ${ }^{30}$

(SAOP)

\section{Multidisciplinary}

assessment of cancer in the ederly ${ }^{31}$ (MACE)

Preoperative Assessment of Cancer in the elderly 32 (PACE)
Programa de oncología para adultos mayores. El programa incluye la evaluación del anciano a través de una valoración geriátrica integral formada por las herramientas: escala de actividades de la vida diaria, actividades instrumentales de la vida diaria, índice de Charlson y escala de puntuación acumulativa de enfermedad en geriatría

Cuestionario multidimensional específico para ancianos con cáncer. Recoge información sobre la demografía, estatus socioeconómico, el estado cognitivo, depresión, rendimiento físico, discapacidad y el estado mental

Evaluación preoperatoria del cáncer en el anciano. Comprende la valoración geriátrica integral de actividades de la vida diaria, actividades instrumentales de la vida diaria, escala de deterioro global, Mini Mental, escala de fragilidad, estado de rendimiento, comorbilidades concurrentes con el índice de comorbilidades de Satarianos (SIC) y el estado físico antes de la cirugía
SAOP comparada con índice de Charlson

Hospitalización Fiabilidad: 0,74 Alfa de Cronbach Sensibilidad: no evaluada Especificidad: no evaluada

MACE comparada con Sickness impact profile

20-45 min Hospitalización Fiabilidad: 0,7 Alfa de Cronbach

Sensibilidad: no evaluada

Especificidad: no evaluada 


\section{Tratamiento}

La decisión terapéutica en los ancianos con cáncer debe considerar que el beneficio sea superior al riesgo que implica ${ }^{2,3}$ y, por supuesto, deben sospesarse las preferencias y deseos del propio anciano. Este punto implica que el anciano esté informado de su diagnóstico y probable pronóstico, pero a menudo hay una retención de la información cuando se transmite el diagnóstico oncológico a ancianos; algunos estudios sugieren que a mayor edad, menos información diagnóstica ${ }^{6,32}$.

El tratamiento elegido debe comprender la curación de la enfermedad, la prolongación de la supervivencia y la paliación de los síntomas ${ }^{6}$. En los ancianos, el tratamiento también tiene la finalidad de mantener la supervivencia activa, es decir, la supervivencia sin pérdida o reducción de la independencia funcional y la calidad de vida ${ }^{2,3}$. La realización de la VGI facilitará la estratificación de estos para la toma final de la decisión terapéutica con el fin de escoger tratamientos con un óptimo perfil de seguridad y eficacia. Actualmente, en España se utiliza la propuesta de Balducci ${ }^{33}$ para la clasificación de los ancianos con cáncer en tres tipos: anciano independiente, anciano parcialmente dependiente y anciano dependiente ${ }^{19-23}$.

El enfoque terapéutico en los ancianos con cáncer debe incluir cirugía, radioterapia, quimioterapia, terapia hormonal y terapias biológicas con agentes diana, y en numerosas ocasiones la asociación entre ellas ${ }^{2,3}$.

La cirugía se considera el tratamiento curativo del cáncer de colon, recto, estómago y páncreas ${ }^{2}$. La función de la EOG es realizar la valoración y seguimiento durante todo el ingreso hospitalario para prevenir e identificar los riesgos y complicaciones más habituales de los ancianos debidos a la cirugía. Dar soporte al equipo asistencial y asesoramiento especializado en intervenciones para prevenir el delirio, el síndrome confusional, la desnutrición, la pérdida funcional, infecciones, retenciones de orina, estreñimiento, dolor, caídas, uso de restricciones e interacciones farmacológicas. También iniciar de forma precoz el alta hospitalaria (programas de educación sanitaria y evaluación del entorno social para poder planificar los recursos más adecuados) $)^{5,6}$.

La radioterapia es una modalidad de tratamiento del que se pueden beneficiar el 60-80\% de ancianos con cáncer; está demostrada su seguridad y eficacia sin asociarse a un aumento de mortalidad si se realiza una planificación y dosimetría correctas para cada individuo, según la localización y el tipo de tumor ${ }^{3}$. También puede combinarse con otras modalidades de tratamiento oncológico como cirugía, quimioterapia, inmunoterapia o tratamiento hormonal. La radioterapia no está exenta de efectos secundarios.

La quimioterapia es el tratamiento sistémico antineoplásico más común. Los cambios fisiológicos que se producen en el organismo debido al envejecimiento interfieren en la farmacocinética, farmacodinamia y la toxicidad del uso de agentes citostáticos ${ }^{3}$. El riesgo de náuseas y vómitos no parecen aumentar con la edad; sí, en cambio, el riesgo de neutropenia, anemia, tronbocitopenia, cardiomiopatía, nefrotoxicidad, neurotoxicidad y mucositis ${ }^{2}$.

La terapia hormonal es el tratamiento de elección para los ancianos, ya que suele ser bien tolerada ${ }^{3}$. Los principales efectos secundarios son sofocos, fatiga, diarreas, náuseas, sensibilidad mamaria, cambios de ánimo, sequedad vaginal, falta de interés por la sexualidad e impotencia ${ }^{2}$.

Las terapias biológicas con agentes diana son aquellas que actúan estimulando o inhibiendo el sistema inmunitario con el fin de tratar el cáncer o los efectos secundarios de otros tratamientos del cáncer ${ }^{3}$. Los principales efectos secundarios de las terapias biológicas son un conjunto de síntomas parecidos a los de la gripe; también puede provocar reacciones de hipersensibilidad grave.
La EOG debe planificar de forma conjunta con el anciano y su familia un plan de autocuidado, realizando un exhaustivo seguimiento de los efectos secundarios esperados, e implementar medidas para tratar las posibles complicaciones. La educación se debe realizar al inicio, durante y en el seguimiento del tratamiento, y debe enseñar al paciente a identificar los signos de alarma y aconsejar las medidas higiénicas y dietéticas que ayudarán a disminuir posibles efectos adversos ${ }^{6}$.

Los ancianos, debido a la polifarmacia, a la complejidad de la pauta posológica y al deterioro funcional y/o cognitivo, pueden incumplir el régimen terapéutico. La no adherencia a los tratamientos se relaciona con peores resultados de salud, peor calidad de vida, mayor probabilidad de recidiva de cáncer, supervivencia más corta y mayor asistencia médi$\mathrm{ca}^{19}$. Las EOG deben detectar aquellos ancianos con riesgo o sospecha de no adherencia e incluirlos en un programa de actuación individualizada, con actividades específicas centradas en favorecer la comprensión de las instrucciones del régimen terapéutico, adaptándolo al estilo de vida y actividades diarias del anciano y familia ${ }^{6}$.

\section{SUPERVIVENCIA}

La Sociedad Española de Oncología Médica (SEOM) define como largo superviviente de cáncer a aquella persona que a los 5 años del diagnóstico y tratamiento se encuentra libre de enfermedad. En España, más del $53 \%$ de los pacientes diagnosticados de cáncer vivirán al menos 5 años $^{16}$. Actualmente no se dispone de guías ni protocolos específicos para la atención del anciano superviviente, pero son diversas las iniciativas y estrategias que se están desarrollando basadas en el seguimiento a largo plazo, el control de la toxicidad diferida o tardía, el manejo de las secuelas físicas y psicológicas, la rehabilitación y la promoción de hábitos saludables ${ }^{34}$. Es muy importante el seguimiento y vigilancia de los efectos tardíos del tratamiento oncológico por parte de la EOG, ya sea a través de consulta o por vía telefónica.

\section{FIN DE VIDA}

Los ancianos con cáncer reciben menor atención de cuidados paliativos al final de vida que los pacientes más jóvenes ${ }^{35}$. La implementación de una atención temprana en cuidados paliativos incluida en el plan integral de cuidados proporciona un mejor control de síntomas, mejora la comunicación y unas expectativas más realistas ${ }^{35}$. La EOG debe ser el nexo entre los múltiples profesionales y las transiciones que configuren su cuidado (hogar, hospital de agudos, rehabilitación, atención primaria, etc.) para asegurar que los planes de atención y objetivos del anciano con cáncer prevalezcan.

\section{DOCENCIA E INVESTIGACIÓN}

Diversos autores han identificado la falta de una formación adecuada estandarizada y reglada en oncología geriátrica ${ }^{1,4,5}$. La formación debe promover habilidades y actitudes que capaciten a las enfermeras para realizar una atención integrada a los ancianos con cáncer para un abordaje multidimensional de sus necesidades. Internacionalmente, esta formación se ha incluido a nivel curricular en formación de posgrado, especialista o formación continua ${ }^{4}$. Integrar el rol investigador en la práctica asistencial de EOG proporcionará amplios beneficios: preservará la utilización de recursos, ofreciendo cuidados basados en la evidencia científica y mejorará la calidad asistencial. Hasta el momento, los estudios de investigación se han centrado en la identificación de herramientas más breves y predictivas, que faciliten la toma de decisiones de tratamientos individualizados ${ }^{22}$. Existen pocos estudios sobre la eficacia 
de las intervenciones o plan de actuaciones integral para el anciano con cáncer; este es un campo de investigación esencial para las EOG ${ }^{36}$. Otra futura área de investigación es el papel del cuidador del anciano con cáncer, ampliamente estudiado en geriatría.

\section{DISCUSIÓN}

Se inició este estudio con el fin de determinar las mejores evidencias científicas en el rol de las enfermeras en el cuidado del anciano con cáncer. Con los resultados encontrados en la literatura científica revisada se puede concluir que faltan ensayos clínicos aleatorizados basados en modelos de atención de enfermería de oncología y geriatría, ya que el mayor número de estudios identificados son revisiones que identificaban los beneficios de incluir la figura de la EOG en el equipo multidisciplinar. En España existen varios proyectos individuales de atención al anciano con cáncer ${ }^{9,24,37}$; uno de ellos incluye la figura de la $\mathrm{EOG}^{24}$, pero no existe una estructura nacional al respecto.

Todos los artículos analizados comparten la importancia de utilizar la VGI como mejor herramienta para la evaluación del anciano con cáncer. En estos últimos meses se ha publicado un documento de recomendaciones de la SEOM sobre la gestión de estos pacientes y que incluye los dominios a evaluar y las escalas que se deben utilizar, coincidiendo con las propuestas en nuestra revisión ${ }^{38}$.

Toda la literatura científica revisada también coincide en que es preciso realizar una atención individualizada, multidimensional y multidisciplinar al anciano con cáncer. Es preciso que esta atención se deba prestar al anciano y su familia durante todo el continuum del cuidado del cáncer, no solamente durante la evaluación, y que incorpore el cuidado de las otras comorbilidades y síndromes geriátricos presentes en el anciano ${ }^{5}$. Una comunicación activa entre los distintos miembros del equipo de atención es clave para evitar la atención fragmentada, el gasto no necesario y resultados no deseados ${ }^{39}$. La EOG es una especialización que requiere formación y educación reglada ${ }^{36,40}$. En la actualidad son múltiples las iniciativas formativas en España, tales como organización de jornadas, congresos y cursos, aunque aún sería preciso incluir esta capacitación y especialización en la formación curricular tanto de enfermeras especialistas en geriatría como especialistas en oncología.

\section{- CONCLUSIÓN}

Es internacionalmente reconocido el papel de las enfermeras como gestoras en la atención a los ancianos con cáncer. El rol de las ECOG debe incluir evaluación, desarrollo, coordinación y seguimiento de planes de actuaciones multidisciplinares y multidimensionales, que pongan en el centro de la atención al paciente y su familia. La EOG debe ser un referente para el paciente, su familia y resto de profesionales, desde su autonomía, para la toma de decisiones complejas. El modelo de cuidado debe abarcar todo el proceso de atención: prevención, detección precoz, evaluación, tratamiento, educación e investigación del anciano con o superviviente de cáncer

\section{Agradecimiento}

Nuestro agradecimiento a todos los profesionales de enfermería y coordinadores asistenciales del Hospital Clínic de Barcelona y del Hospital de Mataró por su apoyo en este proyecto de investigación.

\section{Conflicto de intereses}

Los autores declaran que no existen conflictos de interés.

\section{Financiación}

Los autores confirman no tener ninguna fuente de financiación. Todos los gastos han sido asumidos por los autores.

\section{BIBLIOGRAFÍA}

1. Burhenn PS, McCarthy AL, Begue A, Nightingal G, Cheng K, Kenis C. Geriatric assessment in daily oncology practice for nurses and allied health care professionals: Opinion paper of the Nursing and Allied Health Interest Group of the International Society of Geriatric Oncology (SIOG). J Geriatr Oncol 2016:7(5):315-24.

2. Balducci L. Cáncer y envejecimiento. Tratado de medicina geriátrica. Fundamentos de la atención sanitaria a los mayores. Madrid: Elsevier España; 2015

3. Farré-Mercadé MV Benavent-Boladeras R. Cáncer. Neoplasias más frecuentes. Tratado de geriatría para residentes. Madrid: Sociedad Española de Geriatría y Gerontología; 2006.

4. Burhenn PS, Perrin S, McCarthy AL. Models of care in geriatric oncology nursing. Semin Oncol Nurs. 2016:32(1):24-32.

5. Overcash J. Geriatric Oncology Nursing: Beyond Standard Care Cancer of Aging. 2013;38:139-45.

6. Morgan B, Tarbi E. The role of the advanced practice nurse in geriatric oncology care. Semin Oncol Nurs. 2016;32(1):33-43.

7. Lewis R, Neal RD, Williams NH, France B, Wilkinson C, Hendry M. Nurse-led vs. conventional physician-led follow-up for patients with cancer: Systematic review. J Adv Nurs. 2009;65:706-23.

8. Leeuw J, Larsson M. Nurse-led follow-up care for cancer patients: What is known and what is needed. Support Care Cancer. 2013:21:2643-9.

9. Thomas ML, Elliott JE, Rao SM, Fahey KF, Paul SM, Miaskowsk C. A randomized, clinical trial of education or motivational-interviewing-based coaching compared to usual care to improve cancer pain management. Oncol Nurs Forum. 2012;39(1):39-49

10. Coolbrandt A, Wildiers H, Aertgeerts B, Van der Elst E, Laenen A, Dierckx de Casterlé $B$, et al. Characteristics and effectiveness of complex nursing interventions aimed at reducing symptom burden in adult patients treated with chemotherapy: A systematic review of randomized controlled trials. Int J Nurs Stud. 2014:51:495-510.

11. Cox K, Wilson E. Follow-up for people with cancer: Nurse-led services and telephone interventions. J Adv Nurs. 2003;43:51-61.

12. Reñones Crego MD, Fernández Pérez D, Vena Fernández C, Zamudio Sánchez A. Estrategias para la mejora del cuidado del paciente oncológico: Resultados del proyecto SHARE. Enferm Clin. 2016;26(5):312-20.

13. Agencia de Calidad Sanitaria de Andalucía. Manual de Competencias Profesionales Enfermería de Práctica Avanzada. Enfermero/a de Práctica Avanzada en Cuidados Oncológicos. Consejería de Salud. Enero, 2017.

14. Overcash J, Momever MA. Comprehensive Geriatric Assessment and Caring for the Older Person with Cancer. Semin Oncol Nurs. 2017:33 (4):440-8.

15. Overcash J. Integrating geriatrics into oncology ambulatory care clinics. Clin J Oncol Nurs. 2015;19(4):80-6.

16. Las Cifras del Cáncer en España 2018. Sociedad Española de Oncología Médica (SEOM). Disponible en: https://seom.org/es/noti cias/106525-las-cifras-del-cancer-en-espana-2018

17. Kagan SH, Maloney KW. Cancer Screening and Early Detection in Older People: Considerations for Nursing Practice.Semin Oncol Nurs. 2017:33(2):199-207.

18. Balducci L. Frailty: A common pathway in aging and cancer. Interdiscip Top Gerontol. 2013;38:61-72.

19. Antonio M, Carmona-Bayonas A, Saldaña J, Navarro V, Tebé $C$, Salazar R, et al. Factors Predicting Adherence to a Tailored-Dose Adjuvant Treatment on the Basis of Geriatric Assessment in Elderly People With Colorectal Cancer: A Prospective Study. Clin Colorecta Cancer. 2018:17(1):e59-68.
20. Lynch MP, DeDonato DM, Kutney-Lee A. Geriatric oncology program development and gero-oncology nursing. Semin Oncol Nurs. 2016;32(1):44-54.

21. Saldaña-Cañada J, Yuste- Marco A, Germà-Lluch JR. Importancia de la valoración geriátrica en las personas mayores con cáncer. Rev Esp Geriatr Gerontol. 2010;45(3):121-2.

22. Antonio M, Saldaña J, Formiga F, Lozano A, González-Barboteo J ,

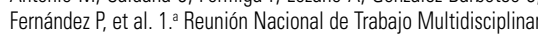
en Oncogeriatría: documento de consenso. Rev Esp Geriatr Gerontol. 2012:47(6):279-283

23. Wildiers $H$, Heeren P, Puts $M$, Topinkova E, Janssen-Heijnen ML, Exterman $\mathrm{M}$, et al. International Society of Geriatric Oncology consensus on geriatric assessment in older patients with cancer. J Clin Oncol. 2014:32(24):2595-603.

24. Molina-Garrido MJ, Guillen-Ponce C. Development of a cancer-specific Comprehensive Geriatric Assessment in a University Hospital in Spain. Crit Rev Oncol Hematol. 2011:77(2):148-61.

25. Luciani A, Ascione G, Bertuzzi C, Marussi D, Codecà C, Di Maria G, et al. Detecting disabilities in older patients with cancer: comparison between comprehensive geriatric assessment and vulnerable elders survey-13. J Clin Oncol. 2010;28(12):2046-50.

26. Soubeyran P, Bellera C, Goyard J, Heitz D, Curé H, Rousselot H, et al. Screening for vulnerability in older cancer patients: the ONCODAGE Prospective Multicenter Cohort Study. PloS One. 2014:9(12):1150-60.

27. Overcash JA, Beckstead J, Moody L, Extermann M, Cobb S. The abbreviated comprehensive geriatric assessment (aCGA) for use in the older cancer patient as a prescreen: scoring and interpretation. Crit Rev Oncol Hematol. 2006;59(3):205-10.

28. Terret C, Albrand G, Moncenix G, Droz JP. Karnofsky performance scale (KPS) or physical performance test (PPT) That is the question? Crit Rev Oncol Hematol. 2011:77(2):142-7. 
29. Baitar A, Van Fraeyenhove F, Vandebroek A, De Droogh E, Galdermans $\mathrm{D}$, Mebis J, et al. Evaluation of the Groningen Frailty Indicator and the G8 questionnaire as screening tools for frailty in older patients with cancer. J Geriatr Oncol. 2013;4(1):32-8.

30. Extermann M, Overcash J, Lyman GH, Parr J, Balducci L. Comorbidity and functional status are independent in older cancer patients. J Clin Oncol. 1998;16(4):1582-7.

31. Monfardini S, Ferrucci L, Fratino L, Del Lungo I, Serraino D, Zagonel V. Validation of a multidimensional evaluation scale for use in elderly cancer patients. Cancer. 1996;77(2):395-401.

32. Pope D, Ramesh H, Gennari R, Corsini G, Maffezzini M, Hoekstra HJ et al. Pre-operative assessment of cancer in the elderly (PACE): a comprehensive assessment of underlying characteristics of elderly cancer patients prior to elective surgery. Surgical Oncol. 2006;15(4):189-97.
32. Font-Ritort $S$, Martos-Gutiérrez JA, Montoro-Lorite $M$, Mundet-Pons L. Calidad de la información sobre el diagnóstico al paciente oncológico terminal. Enferm Clin. 2016;26(6):344-50

33. Balducci L. Recomendaciones para el tratamiento del cáncer en el anciano: implicaciones para la calidad de vida. Rev Esp Geriatr Gerontol. 2004;39(4):270-6.

34. Sociedad Española de Oncología Médica. Manifiesto SEOM sobre la Atención a los Largos Supervivientes de Cáncer. Disponible en: https://seom.org/publicaciones/publicaciones-seom/largos-supervivientes

35. Brighi N, Balducci L, Biasco G. Cáncer en el anciano: es tiempo para los cuidados paliativos en oncología geriátrica? J Geriatr Oncol. 2014;5:197-203.
36. Van Cleave JH, Kenis C, Sattar S, Jabloo VG, Ayala AP, Puts M. A research agenda for gero-oncology nursing. Semin Oncol Nurs. 2016:32(1):55-64.

37. Molina-Garrido MJ. Implementación de una consulta de cáncer en el anciano. Innovación a la atención en el paciente oncológico. Disponible en: http://www.innovonco.es/pdf/testimonial-1.pdf

38. Sarrió RG, Rebollo MA, Garrido MM, Guillén-Ponce C, Blanco R, Flores $E G$, et al. General recommendations paper on the management of older patients with cancer: the SEOM geriatric oncology task force's position statement. Clin Transl Oncol. 2018:20(10):1246-51.

39. Shahrokni A, Kim SJ, BosI GJ, Korc-Grodzicki B. How we care for an older patient with cancer. J Oncol Pract. 2017;13(2):95-102

40. Hsu T. Educational initiatives in geriatric oncology-Who, why, and how? J Geriatr Oncol. 2016;7(5):390-6. 